\title{
The Optimal Choice of Pre-Launch Reviewer
}

\author{
David Gill \\ Trinity College and Dept. of Economics \\ University of Oxford
}

\author{
Daniel Sgroi ${ }^{1}$ \\ Faculty of Economics and Churchill College \\ University of Cambridge
}

This Version: 12th November 2006

\begin{abstract}
We consider the impact of pre-launch reviews on sales of products of quality unknown to consumers. Sales occur simultaneously after consideration by a reviewer with a known level of bias. Consumers observe the reviewer's decision and a private signal. Using convex analysis we prove three major results: (i) a monopolist, which can choose the type of reviewer and the price conditional on the chosen reviewer's decision, will always want to have its product reviewed; (ii) choosing an extreme reviewer, that is either the toughest or softest reviewer type available, is always optimal; and (iii) the switch from the toughest to softest reviewer occurs when the precision of private information reaches a calculable threshold. The paper provides a justification for the existence of heavily biased reviews and tests, and also suggests a clear course of action for firms in different situations. For example, an entrant with a very innovative product should risk a tough pre-launch test, while an incumbent with a new product that provides only a small change over an existing one would do best using a soft pre-launch review.
\end{abstract}

Keywords: reviewers, Bayesian learning, bias, marketing

JEL classification: D82, D83, L15

1 Daniel Sgroi can be contacted at daniel.sgroi@econ.cam.ac.uk and David Gill can be contacted at david.gill@economics.ox.ac.uk. Both authors would like to thank the Economic and Social Research Council for financial support, and to thank participants at seminars in Birkbeck, Cambridge, Exeter and Oxford, and at the Second World Congress of the Game Theory Society in Marseille (2004), the Econometric Society World Congress in London (2005), the ESRC Game Theory Conference in Essex (2005) and the European Economic Association Annual Congress in Vienna (2006) for helpful comments and suggestions. 


\section{Introduction}

Reviewers can be incredibly powerful. For example, in the wine trade the American reviewer Robert Parker can make or break a new vintage. According to the Oxford Companion to Wine: "His judgements have had a significant effect on market demand and the commercial future of some producers... [for example] he was instrumental in establishing the reputation and ambitious pricing policy of Côte Rôtie's Guigal." (Robinson, 1999, pp. 511-512). Despite the powerful effects that reviewers can exert over new products launched onto the market, the literature has paid little or no attention to the use of public reviews. This article attempts to correct the omission.

When a firm develops a new product, it will often consider where to send it for pre-launch review or accreditation. Our model applies to situations in which the firm can choose from a range of pre-launch reviewers or tests of varying toughness or bias, and where the firm can select an optimal price based on the reviewer's report. The choice of reviewer involves an inherent trade-off. Tough reviewers are unlikely to endorse the product, but they generate a strong positive impact on sales and prices if they do endorse, while a failure to endorse is not too damaging as everybody understands that their standards are high. Soft reviewers, on the other hand, exhibit the opposite characteristics: they are likely to endorse, but the impact of endorsement is weak, and a failure to endorse can be catastrophic.

Consider, for example, the publisher of a new computer game or piece of software. The firm could opt to send early "beta" versions of the product to pre-launch reviewers for a preview. Should it do so, it can select which of a range of journals and online sites are allowed access to a preview copy of the software. Sites and journals typically have a known toughness. For example, general video games websites will often list not only the results of reviews and previews, but also give some indication of the reviewer's toughness, while "official" games magazines owned by the same company that produces a game might be considered "soft" in the sense of Definition 1 below. The software firm's decision therefore boils down to deciding whether a pre-launch reviewer should be approached, and if so, what level of toughness to select. Early success for software in a preview can have huge implications for the sales of the product, especially in the pre-order market, where games in particular are pre-purchased by consumers before general release. The findings in the pre-launch review can also help the software publisher choose an appropriate price.

In a different setting, a Ph.D. student entering the job market may have a similar choice 
between using well-known "soft" or "tough" professors as referees. A strong reference from a well-respected tough professor can mean a shot at one of the top universities or business schools and hence a higher salary. Similar logic might influence where a film-maker sends his latest movie to premiere: to a famous film festival, where competition for prizes is tough, in an effort to win international acclaim, or somewhere with less difficult competition to overcome. Assuming the film is accepted for screening, success or failure in winning a prize at a particular festival will then influence the contractual terms offered to exhibitors. ${ }^{2}$ Other examples include technology sponsors who can choose between different standard setting organizations ${ }^{3}$, issuers of stocks and bonds who can attempt "to receive certification from investment banks, analysts, and rating agencies, which differ in their reputation and independence" (Lerner and Tirole, 2006, p. 1091), and restaurantowners who can choose which critics to invite to their opening night.

In our model, a monopolist is launching a product which is of either high or low quality. Quality is known to the firm, but not to the consumers, and is unverifiable. Consumers' information about quality comes from two sources. First, they receive a private signal. If the firm chooses to have its product reviewed or tested, they also observe the public reviewer's report or test result. Reviewers are assumed to be better informed than consumers, modelled by receiving an additional signal. As the reviewers receive multiple signals, they will often face mixed evidence of a product's quality. We vary the probability of endorsing on this mixed set of signals - the probability of endorsement measures the reviewer's toughness, and is common knowledge. Having observed the type of reviewer selected by the firm, the reviewer's report, the price chosen after the report becomes known and their private signals, the consumers simultaneously decide whether or not to purchase.

In this context, a firm with a low quality product can costlessly duplicate the actions of a firm with a high quality product. As a result, all of our outcomes will be pooling, and there will be no issues of incentive compatibility or scope for a separating equilibrium - the firm cannot use its choice of type of pre-launch review to directly signal the quality of the product. Nevertheless, reviewers play an important role in information revelation. We find that the firm will always want

2 De Vany and Walls (1996) offer a good summary of these terms. The fixed fee charged to the exhibitor and the minimum run period are both negotiable, as are the advance distribution fees agreed with the post-theatrical market such as pay-TV.

3 As Lerner and Tirole (2006, p.1091) put it: "Technology sponsors attempt to build standards around their technologies by having them validated by standard setting organizations (SSOs) that range from fully independent to largely captive special interest groups (SIGs)." 
to have its product reviewed and will choose an extreme reviewer, selecting either the toughest or softest reviewer type available. Where consumers have no prior information about quality and the signals received by the consumers and reviewer are of low precision, which might correspond to a new firm or type of product, the firm will choose the toughest reviewer available, maximizing the impact of an endorsement. The firm hopes to launch its product with a bang. If on the other hand signals are of high enough precision, perhaps because the firm or product type is well-known, the softest reviewer is chosen, maximizing the probability of endorsement.

The article proceeds as follows. The next section reviews the related literature. Section 3 sets up the model. Section 4 justifies the restriction to pooling equilibria. Section 5 analyzes the choice of optimal pre-launch reviewer and conditional price. Section 6 concludes.

\section{Related Literature}

No extant article combines the choice of reviewer and price for a firm as we do; however there are various related literatures.

A number of articles analyze the use of initial prices to manipulate sales in a learning environment. For example, Taylor (1999) and Bose, Orosel, Ottaviani and Vesterlund (2005a, 2005b) find that high initial prices, whose effects are similar to a choice of tough reviewer, can be optimal. In Bose et al., the firm (which unlike in our model does not know the quality of its own product) wishes to set a high initial price (relative to perceived quality) to encourage the transmission of information. If price is too low, everybody buys, so consumers do not learn from each other's decisions, while if an expensive good becomes successful (the analogue of an endorsement from a tough reviewer), this conveys strong positive information to later buyers. Taylor, concentrating on the housing market, finds a high price to be optimal as a failure to sell a house early (the analogue of a tough reviewer failing to endorse) can then be attributed to overpricing rather than low quality. By contrast, in Caminal and Vives $(1996,1999)$, in which early prices are unobservable to later consumers, and in Welch (1992), in which prices cannot be conditioned on the history of purchases, low introductory prices are optimal.

Lerner and Tirole's (2006) recent (and independently developed) paper concerns the role of technology standard setting authorities as certifiers. ${ }^{4}$ Similarly to our reviewers, the certifiers

\footnotetext{
4 Chiao, Lerner and Tirole (2005) empirically test Lerner and Tirole's model, while Farhi, Lerner and Tirole (2005)
} 
have an arbitrary bias towards the technology sponsor which determines their endorsement rule. The model has significant differences to ours: the sponsor is not perfectly informed about the quality of its technology; the chosen certifier discovers with certainty the quality of the technology it is asked to review; consumers do not receive any private information; and there is no incentive to set price in response to the certification (as the certifier's endorsement rule is sensitive to any anticipated price response to its decision). Therefore, as certifiers cannot counter bad private information or enable a rise in price, Lerner and Tirole do not find any role for certifiers biased against the technology. Instead they find that the sponsor prefers the certifier most biased in favor of the new technology on offer, subject to users adopting following an endorsement. This is in stark contrast to our findings, which allow a role for reviewers biased in either direction depending upon the quality of private information.

Our paper should be contrasted with the literature on experts, in which self-interested experts filter information about the true state of the world (see chapter 10 of Chamley (2004) for a survey). Their self-interest gives rise to incentives to manipulate the messages they send. We, on the other hand, assume that our reviewers have no self-interested motives, apart from taking on biases to appeal to firms. Our work is also different from the literature on payment structures to certification intermediaries - see for example Lizzeri (1999) and Albano and Lizzeri (2001). The question there is how intermediaries affect the quality chosen by the firm, while in our model quality is fixed. Disclosure may turn out to be incomplete, but it's not allowed to be biased.

We assume flexible prices, but in some settings, prices may not be variable conditional on the reviewer's decision. In Gill and Sgroi $(2003,2006)$ we consider a sequential sales model with pre-launch tests but without prices. As the length of the sequence of consumers tends to one, that model can be re-interpreted as the analogue of the simultaneous sales model in this paper, but with fixed prices. We find that a tough test is always preferred. When prices cannot be adjusted in light of the test/review, passing a tough test swamps bad private information, while a failure to pass a tough test is not enough to damage good private information, so tough tests are valuable. Where available, the firm will choose a tough test that is very close to unbiased to maximize the chance of passing. In Sgroi (2002) multiple public decisions made by consumers at the start of a product's life-cycle act in a similar way to a reviewer, and one of the key results is that a firm extend the model to a dynamic setting. 
should optimally select to use such early decision makers to boost profits. While this provides some support for the result that a pre-launch reviewer will increase profits, the paper has neither prices nor a notion of bias, so can offer no guide as to the optimal toughness of such a reviewer.

\section{The Model}

Consider a group of $N \in \mathbb{N}_{++}$risk-neutral consumers who act simultaneously deciding whether to purchase or not purchase some product of unverifiable high or low quality $V \in\{0,1\} .^{5}$ The price of purchase is $\lambda$, which results in a net gain of $V-\lambda$. The utility from not purchasing is 0 . The prior probability that the good is of high quality $(V=1)$ is $q \in(0,1)$. The risk-neutral monopolist knows the quality of its product, while the agents each receive a conditionally (on $V$ ) independent signal about $V$ defined as $X_{i} \in\{H, L\}$ for agent $i$ drawn from a Bernoulli distribution with parameter $p{ }^{6}$ The signals are informative but not fully revealing in the sense that:

$$
\begin{aligned}
& \operatorname{Pr}\left[X_{i}=H \mid V=1\right]=\operatorname{Pr}\left[X_{i}=L \mid V=0\right]=p \in\left(\frac{1}{2}, 1\right) \\
& \operatorname{Pr}\left[X_{i}=H \mid V=0\right]=\operatorname{Pr}\left[X_{i}=L \mid V=1\right]=1-p \in\left(0, \frac{1}{2}\right)
\end{aligned}
$$

Before launching its product onto the market, the firm is able to have it publicly reviewed or tested. We want to think of reviewers as having access to finer information because the firm allows the reviewer to test the product. The simplest way of modelling this is to allow the reviewer to receive two i.i.d. draws from the same signal distribution as consumers. Reviewers make a binary decision $d \in\{E, F\}$ whether to endorse $(E)$ or fail to endorse $(F)$ the firm's product. (In the case of a test, we can think of $E$ being a pass, while $F$ is a fail.). Modelling an evaluator as condensing more complex information into a simple binary decision follows for example Calvert (1985) and Sah and Stiglitz (1986). As Calvert (p. 534) puts it: "This feature represents the basic nature of advice, a distillation of complex reality into a simple recommendation." Reviewers endorse if they observe $H H$, endorse with probability $\phi \in[0,1]$ if they observe $H L$ or $L H$ and fail to endorse if

5 Propositions 1 and 2 extend straightforwardly to the more general case where $V \in\{\underline{V}, \bar{V}\}$ and there is a constant marginal cost of production $c$ so long as $0 \leq c \leq \underline{V}<\bar{V}$. Appendix $\mathrm{C}$ demonstrates this.

6 In a setting where both the buyer and seller are uninformed about quality, Ottaviani and Prat (2001) also find that a monopolist may wish to use a public signal of quality such as an outside certifier. In their model, a public signal affiliated with the buyer's private information reduces the buyer's informational rents in a second-degree price discrimination setting. 
they observe $L L$. The value of $\phi$ encapsulates the type of the reviewer. The lower the value of $\phi$, the tougher the test that is imposed. ${ }^{7}$

Definition 1 Reviewers with $\phi \in\left[0, \frac{1}{2}\right)$ are termed "pessimistic" (tough), and those with $\phi \in\left(\frac{1}{2}, 1\right]$ "optimistic" (soft, easy). When $\phi=1$ the reviewer is an "extreme optimist", who endorses having seen any combination of signals from $\{H H, H L, L H\} . \phi=\frac{1}{2}$ corresponds to an "unbiased" (neutral) reviewer who flips a fair coin on observing a set of mixed signals. $\phi=0$ corresponds to an "extreme pessimist" who only endorses when $H H$ is observed.

Reviewer types are common knowledge, i.e., we are assuming that the firm and consumers can discover a reviewer's type from previous endorsement decisions and other public information. ${ }^{8}$ If consumers did not know the reviewers' types, clearly the firm would choose the softest reviewer available (perhaps one financially supported or wholly owned by the firm), and be expected to do so by consumers.

The firm chooses a reviewer of type $\phi$ from the continuum $[0,1] .{ }^{9}$ Once the reviewer's decision is made public, the firm chooses a profit-maximizing price $\lambda$, which can be conditioned on both the reviewer's type and endorsement decision. Consumers update their beliefs based on their private information, on the reviewer's type and decision, and on the chosen price $\lambda$, and purchase if $E\left[V \mid \phi, d, X_{i}, \lambda\right] \geq \lambda .{ }^{10}$ We restrict the firm to pure strategies.

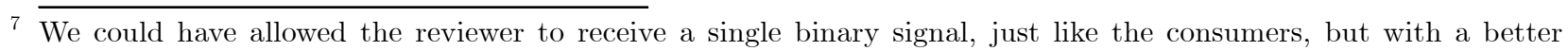
quality of information $p_{R}>p$. Bias could then be measured by the probability of endorsement $\alpha \in[0,1]$ when receiving a $H$ signal in the case of a tough reviewer, and $\beta \in[0,1]$ when receiving a $L$ signal in the case of a soft reviewer. However, such an analysis holds little interest, as then conditional on endorsement by a tough reviewer, beliefs about quality are not changing in $\alpha$ as consumers infer that the reviewer definitely saw a $H$ signal. This holds true even if $\alpha$ rises as high as 1 , giving an unbiased reviewer. Thus we lose the intuitively appealing feature that the tougher the reviewer, the better the signal from an endorsement, and we do not get the benefit of a higher price conditional on endorsement as the reviewer becomes tougher. Similarly, conditional on a failure to endorse by a soft reviewer, beliefs are not affected by $\beta$, as a $L$ signal is inferred from a failure to endorse. In our model, the reviewer is effectively receiving a trinary signal, so the binary report is coarse relative to the signal space. It is this coarseness which gives bias its purchase in our results. Though beyond the scope of this paper, we conjecture that our results are robust to more nuanced reviewer reports and signals, so long as the report is coarser than the original signal.

8 Note that reviewers are assumed to be able to build up a reputation without consumers having to purchase or discover product quality. Given the prior expectation of product quality, consumers will be able to estimate a reviewer's type from a history of endorsement decisions.

9 By allowing the firm to select just one reviewer, we are abstracting from the use of multiple reviewers to advertise the existence or availability of the product.

${ }^{10}$ The purchase at indifference rule is without loss of generality, as if at a given price consumers who were indifferent purchased with probability less than one, the firm could always sell to all the indifferent consumers by shaving price by a small amount $\epsilon>0$. 


\section{Restriction to Pooling Equilibria}

Throughout, we consider just the preference of the firm with a good product. A bad product firm can costlessly duplicate the good product firm's choice of $\{\phi, \lambda\}$, so a separating equilibrium is not possible: the bad product firm would copy the choice of the good product firm, and so be believed to be good and make the same profit as the good product firm (selling to all consumers at a price $\lambda$ ). Thus, we restrict attention to pooling equilibria in which the bad product firm is forced to follow the good product firm's preferred choice of $\{\phi, \lambda\}$ (conditional on being in a pooling equilibrium) to avoid immediately revealing its type and so get zero sales. Such equilibria can always be supported by the belief that any firm which deviates from the good product firm's preferences is selling a low quality product. ${ }^{11}$ Note that in such pooling equilibria, consumers will be unable to infer anything about product quality from the firm's choice of reviewer or price, and hence the statement in the introduction that this cannot reasonably be considered a signalling paper. ${ }^{12}$ Furthermore, because we have assumed that product quality is non-verifiable, our model is not a game of persuasion à la Milgrom (1981), so firms are unable to directly reveal information to try to separate from their rivals, and nor can guarantees be used.

\section{The Optimal Choice of Reviewer and Price}

In this section, we determine the firm's optimal choice of pre-launch reviewer and profit-maximizing price conditional on the chosen reviewer's report. As explained in the previous section, we focus on the good product firm's choice. The first subsection shows that extreme reviewers are optimal, the second that the firm will want to have its product reviewed, while the third considers which extreme the firm will want to select.

${ }_{11}$ Of course, pooling on any arbitrary $\{\phi, \lambda\}$ can also be supported by such beliefs, but we do not believe such equilibria to be reasonable. Appendix A discusses this in detail. Semi-separating equilibria are ruled out by our restriction to pure strategy equilibria.

12 Judd and Riordan (1994) find that a firm launching a new product whose quality it does not know perfectly may be able to use price as a signalling device. Where the firm does know quality, Mahenc (2004) also finds that separation via price is possible, but only if some consumers are perfectly informed. 


\subsection{Optimality of Extreme Reviewers}

Let $\mu_{d, X_{i}}=\operatorname{Pr}\left[V=1 \mid d, X_{i}\right]$ where the reviewer's decision $d \in\{E, F\}$ and the consumer's signal $X_{i} \in\{H, L\}$. Using Bayes' Rule:

$$
\mu_{d, X_{i}}=\frac{\operatorname{Pr}\left[d, X_{i} \mid V=1\right] q}{\operatorname{Pr}\left[d, X_{i} \mid V=1\right] q+\operatorname{Pr}\left[d, X_{i} \mid V=0\right](1-q)}
$$

After the reviewer's decision, the firm will choose to set price $\lambda=\mu_{d, H}$ to sell to all those who received high private signals or $\lambda=\mu_{d, L}$ to sell to all consumers including those with low private signals. Any other price would be sub-optimal. With $\lambda>\mu_{d, H}$ no consumers will buy, with $\lambda \in\left(\mu_{d, L}, \mu_{d, H}\right)$ only the high signal consumers will buy at a price lower than $\mu_{d, H}$, and with $\lambda<\mu_{d, L}$ everybody buys at a price lower than $\mu_{d, L}$.

Without loss of generality, we now normalize the number of customers to 1 , so expected profits as a function of these prices are $\pi_{d}\left(\mu_{d, H}\right)=p \mu_{d, H}$ and $\pi_{d}\left(\mu_{d, L}\right)=\mu_{d, L}$. We define $\pi_{d}^{*}=\max \left\{p \mu_{d, H}, \mu_{d, L}\right\}$ to be the maximum expected profits achievable given the reviewer's decision, which will be a function of $p, \phi$ and $q$. Then the firm's optimization problem reduces to choosing the reviewer type $\phi$ to maximize ex ante expected profits $\Pi$ :

$$
\max _{\phi \in[0,1]} \Pi=\left[\operatorname{Pr}[E] \pi_{E}^{*}+\operatorname{Pr}[F] \pi_{F}^{*}\right]
$$

We begin by showing that $\Pi$ is strictly convex in $\phi$, which implies that the firm will always choose an extreme type, choosing either an extreme pessimist with $\phi=0$ or an extreme optimist with $\phi=1$. We then move on to show which extreme is best over the range of $p$. First, we note the following crucial lemma.

Lemma $1 \frac{d^{2}\left(\operatorname{Pr}[d] p \mu_{d, H}\right)}{d \phi^{2}}>0$ and $\frac{d^{2}\left(\operatorname{Pr}[d] \mu_{d, L}\right)}{d \phi^{2}}>0$ for $d \in\{E, F\}$.

Proof. See Appendix B.

Now,

$$
\begin{aligned}
\Pi & =\left[\operatorname{Pr}[E] \pi_{E}^{*}+\operatorname{Pr}[F] \pi_{F}^{*}\right]=\operatorname{Pr}[E] \max \left\{p \mu_{E, H}, \mu_{E, L}\right\}+\operatorname{Pr}[F] \max \left\{p \mu_{F, H}, \mu_{F, L}\right\} \\
& =\max \left\{\operatorname{Pr}[E] p \mu_{E, H}, \operatorname{Pr}[E] \mu_{E, L}\right\}+\max \left\{\operatorname{Pr}[F] p \mu_{F, H}, \operatorname{Pr}[F] \mu_{F, L}\right\}
\end{aligned}
$$


Thus using Lemma 1, the fact that the maximum of a set of strictly convex functions all defined over the same range must itself be strictly convex (see Rockafellar, 1970), and the fact that the sum of strictly convex functions is also strictly convex, $\Pi$ must be strictly convex and Proposition 1 follows. ${ }^{13}$

Proposition 1 The good product firm always strictly prefers either the extreme pessimist $(\phi=0)$ or the extreme optimist ( $\phi=1)$, or both, to any intermediate reviewer with $\phi \in(0,1)$.

To provide an intuition for the proposition, first note that all prices $\left\{\mu_{E, H}, \mu_{E, L}, \mu_{F, H}, \mu_{F, L}\right\}$ are decreasing in $\phi$ : an endorsement raises beliefs more the tougher the reviewer, while a failure to endorse is not so damaging to beliefs if the reviewer is tougher. Furthermore, $\mu_{E, H}$ and $\mu_{E, L}$ can be shown to be strictly convex in $\phi$, so conditional on endorsement a decrease in $\phi$ is more powerful where the reviewer is tougher. This can be seen by noting that from the consumers' perspective the relative probability of the reviewer having seen two good signals to having seen mixed signals is $\frac{p^{2} q+(1-p)^{2}(1-q)}{2 p(1-p) \phi}$, which is convex in $\phi .{ }^{14}$ On the other hand, $\operatorname{Pr}[E]$ is linearly increasing in $\phi$. Thus, to maximize $\operatorname{Pr}[E] p \mu_{E, H}$ or $\operatorname{Pr}[E] \mu_{E, L}$, the firm will wish to choose an extreme $\phi$, either setting $\phi=0$ to benefit from a steep increase in price while the probability of endorsement falls linearly, or setting $\phi=1$ to benefit from a linear increase in the probability of endorsement, while price does not fall much towards the end.

To maximize $\operatorname{Pr}[F] p \mu_{F, H}$ or $\operatorname{Pr}[F] \mu_{F, L}$, the firm will always want to set $\phi=0$, as both $\operatorname{Pr}[F]$ and the prices are decreasing in $\phi$. The proof of Lemma 1 shows that $\operatorname{Pr}[F] p \mu_{F, H}$ and $\operatorname{Pr}[F] \mu_{F, L}$ are also convex. Thus, summing over the case of endorsement and failure to endorse, the firm always prefers an extreme $\phi$. The firm will either choose the extreme pessimist to maximize prices, at the cost of a lower probability of endorsement, or the extreme optimist to maximize the probability of endorsement, at the cost of lower prices.

Of further interest, and a direct implication of the convexity of $\Pi$, is the following remark.

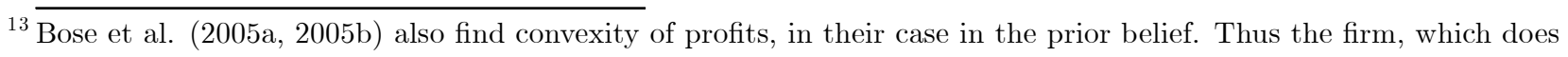
not know its own product quality, will want to release information - setting a high price to early customers reveals information about their private signals, moving the prior up or down, while at a low price everybody buys so nothing is revealed. In our model, the firm knows its product quality, and the good product firm clearly wants to release as much information as possible. The convexity of profits in $\phi$ shows that information revelation is best achieved using an extreme reviewer.

${ }^{14}$ As $\operatorname{Pr}[H H \mid E]=\frac{\operatorname{Pr}[E \mid H H] \operatorname{Pr}[H H]}{\operatorname{Pr}[E]}=\frac{p^{2} q+(1-p)^{2}(1-q)}{\operatorname{Pr}[E]}$ and $\operatorname{Pr}[\{H L$ or $L H\} \mid E]=\frac{\operatorname{Pr}[E \mid\{H L \text { or } L H\}] \operatorname{Pr}[\{H L \text { or } L H\}]}{\operatorname{Pr}[E]}=$ $\frac{\phi[2 p(1-p) q+2 p(1-p)(1-q)]}{\operatorname{Pr}[E]}$. 
Remark 1 For any finite set of $\phi$ values of size $K>2$ such that $\phi_{1}>\phi_{2}>\ldots>\phi_{K}$, $\phi_{1}$ or $\phi_{K}$ would always be strictly preferred by the good product firm to any intermediate reviewer with $\phi_{j} \notin\left\{\phi_{1}, \phi_{K}\right\}$.

So even with a restricted choice range, extremists are preferred. In the limit with only three reviewer types, an unbiased, a tough and a soft, we know that the best choice cannot be the unbiased.

\subsection{Optimality of Reviews}

Proposition 1 shows that from the set of reviewers, an extremist will always be preferred, but the firm could of course opt not to have its product reviewed at all. We now show that the firm will always want to undergo a review. Let $\mu_{X_{i}}=\operatorname{Pr}\left[V=1 \mid X_{i}\right]$. Using Bayes' Rule:

$$
\mu_{X_{i}}=\frac{\operatorname{Pr}\left[X_{i} \mid V=1\right] q}{\operatorname{Pr}\left[X_{i} \mid V=1\right] q+\operatorname{Pr}\left[X_{i} \mid V=0\right](1-q)}
$$

Where the firm does not use a reviewer, it will (similarly to the case with a reviewer) set price $\lambda=\mu_{H}$ to sell to all those who received high private signals or $\lambda=\mu_{L}$ to sell to all consumers including those with low private signals. Once again normalizing the number of customers to 1 , expected profits as a function of these prices are $\pi\left(\mu_{H}\right)=p \mu_{H}$ and $\pi\left(\mu_{L}\right)=\mu_{L}$. We define $\Pi($ No Reviewer $)=\max \left\{p \mu_{H}, \mu_{L}\right\}$ to be the maximum expected profits achievable without a reviewer.

Let $\Pi_{H H}=\operatorname{Pr}[E] p \mu_{E, H}+\operatorname{Pr}[F] p \mu_{F, H}$ and $\Pi_{L L}=\operatorname{Pr}[E] \mu_{E, L}+\operatorname{Pr}[F] \mu_{F, L}$. For a given $\phi$, these represent the profits to a firm which decides before seeing the reviewer's decision whether to set a high price to sell only to high private signal consumers or a low price to sell to everyone. Note that such a firm will still condition price on the reviewer's decision, as $\mu_{E, H}>\mu_{F, H}$ and $\mu_{E, L}>\mu_{F, L}$. Then the following lemma holds.

Lemma $2 \Pi_{H H}\left(\phi=\frac{1}{2}\right)>p \mu_{H}$ and $\Pi_{L L}\left(\phi=\frac{1}{2}\right)>\mu_{L}$.

Proof. See Appendix B.

Thus for any $p \in\left(\frac{1}{2}, 1\right), \max \left\{\Pi_{H H}\left(\phi=\frac{1}{2}\right), \Pi_{L L}\left(\phi=\frac{1}{2}\right)\right\}>\max \left\{p \mu_{H}, \mu_{L}\right\}$. Remember that $\Pi=\operatorname{Pr}[E] \max \left\{p \mu_{E, H}, \mu_{E, L}\right\}+\operatorname{Pr}[F] \max \left\{p \mu_{F, H}, \mu_{F, L}\right\}$ is the maximum expected profit 
achievable for a given $\phi$ when the firm is able to fully condition price on the reviewer's decision. Clearly, $\Pi \geq \max \left\{\Pi_{H H}, \Pi_{L L}\right\}$ as a firm which is able to condition the decision about whether to set the high or low price on the reviewer's decision must do weakly better than one which does not do so. Thus, we can conclude that $\Pi\left(\phi=\frac{1}{2}\right) \geq \max \left\{\Pi_{H H}\left(\phi=\frac{1}{2}\right), \Pi_{L L}\left(\phi=\frac{1}{2}\right)\right\}>$ $\max \left\{p \mu_{H}, \mu_{L}\right\}=\Pi$ (No Reviewer), which proves the following proposition.

Proposition 2 The choice of no reviewer is strictly worse than the unbiased reviewer type $\phi=\frac{1}{2}$ and a fortiori strictly worse than the optimal reviewer.

The intuition here is that because the firm can alter price in response to the unbiased reviewer's decision, even choosing an unbiased reviewer is better than choosing no reviewer at all.

\subsection{The Optimal Extreme}

Figure 1 shows which extreme is preferred for general prior $q$ and precision of private signals $p$. When the plot is above zero, the firm prefers the extreme optimist, and when it is below zero the preference is for an extreme pessimist. It is apparent from this diagram that the choice of extreme is non-trivial. However, we observe that for low $q$, as signal precision rises the firm moves from preferring the extreme pessimist to preferring the extreme optimist, while for high $q$, the firm's preference moves the other way.

Figure 1: $\Pi(\phi=1)-\Pi(\phi=0)$

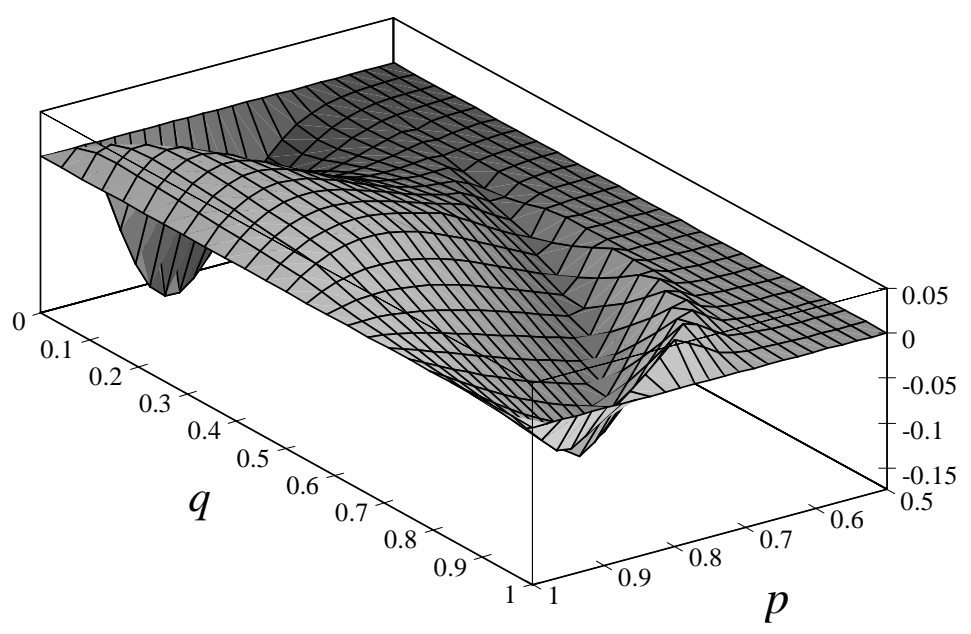


Henceforth, we study the problem analytically for the case where consumers' priors are uninformative $\left(q=\frac{1}{2}\right)$, so all consumer information comes from their signals and the reviewer's decision. This keeps the analysis simple, but we also believe that thinking of the consumers as uninformed about quality until they start receiving noisy signals is a good model of the world. We start by finding $\pi_{d}^{*}$ for $\phi=1$ and $\phi=0$.

Lemma 3 With uninformative priors:

(i) Following a failure to endorse, and with $\phi=1, \mu_{F, L}$ is superior for $p \in\left(\frac{1}{2}, p_{1}\right)$ and $p \mu_{F, H}$ is superior for $p \in\left[p_{1}, 1\right)$, where $p_{1} \simeq 0.594$.

(ii) Following a failure to endorse, and with $\phi=0, \mu_{F, L}$ is superior for $p \in\left(\frac{1}{2}, p_{2}\right)$ and $p \mu_{F, H}$ is superior for $p \in\left[p_{2}, 1\right)$, where $p_{2} \simeq 0.607$.

(iii) Following endorsement, and with $\phi=1, \mu_{E, L}$ is superior for $p \in\left(\frac{1}{2}, p_{3}\right)$ and $p \mu_{E, H}$ is superior for $p \in\left[p_{3}, 1\right)$, where $p_{3} \simeq 0.635$.

(iv) Following endorsement, and with $\phi=0, \mu_{E, L}$ is superior $\forall p \in\left(\frac{1}{2}, 1\right)$.

Proof. See Appendix B.

The negative effect of rejection is stronger for higher $p$, and as $p$ goes up, the proportion of consumers with positive private signals increases. Thus, for $p<p_{1}$ the firm sells to everybody following a rejection, as the failure to endorse is not too damaging and relatively few consumers receive high private signals, but for $p \geq p_{2}$, the firm focuses on just those with high signals. In a small range $p \in\left[p_{1}, p_{2}\right)$, the degree of bias affects the choice: the firm sells to everybody if and only if the reviewer is pessimistic, as rejection then sends a weaker signal. The positive signal arising from an endorsement by an extreme pessimist is strong enough that the firm wants to sell to everybody for all $p$. Endorsement by an extreme optimist sends a much weaker signal, so for $p \geq p_{3}$ the proportion of high signal consumers and the price that the firm can charge them are high enough that the firm sells just to them. We can now determine whether the extreme pessimist or optimist is superior for any given $p$.

Remark 2 With uninformative priors, the good product firm prefers the extreme pessimist for $p \in\left(\frac{1}{2}, p_{4}\right)$, while the extreme optimist is preferred for $p \in\left[p_{4}, 1\right)$, where $p_{4}=-1+\sqrt{3} \simeq 0.732$. The preferences are strict, except at $p_{2}$ and $p_{4}$, where the firm is indifferent between the two extremes. 
Proof. See Appendix B.

The extreme pessimist is best for lower values of $p$ while for higher values the extreme optimist is best. This is by no means obvious given the complex interaction of various functions of $p$, but with the assistance of some diagrams the optimal choice of extremist becomes apparent. In Figure 2 , the two continuously increasing lines show $\operatorname{Pr}[E] \pi_{E}^{*}$ evaluated over the range of $p$, the higher line for $\phi=1$ and the lower line for $\phi=0$.

\section{Figure 2}

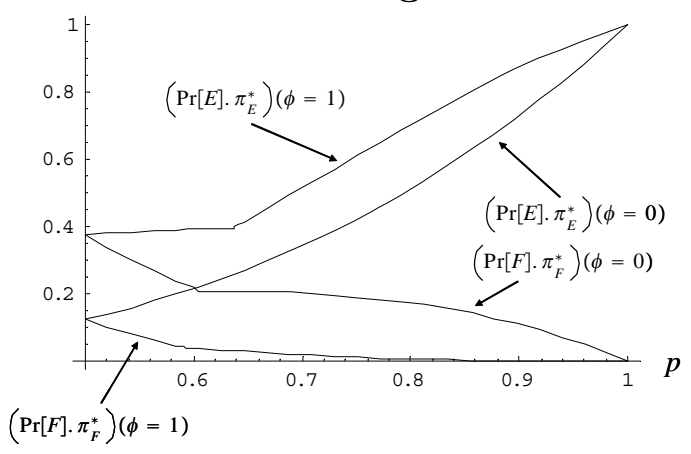

We can see that $\operatorname{Pr}[E] \pi_{E}^{*}$ is always higher with the extreme optimist: the higher probability of endorsement overwhelms the lower price. The two decreasing lines show $\operatorname{Pr}[F] \pi_{F}^{*}$, the higher line for $\phi=0$ and the lower line for $\phi=1$. In contrast to the endorsement case, $\operatorname{Pr}[F] \pi_{F}^{*}$ is always higher under the extreme pessimist, as both prices and $\operatorname{Pr}[F]$ are higher. The kinks in the curves represent values of $p$ at which the firm moves from setting a low price to sell to everybody, to a high price to sell only to those who receive high private signals.

Given $p$, the firm wants to maximize $\operatorname{Pr}[E] \pi_{E}^{*}+\operatorname{Pr}[F] \pi_{F}^{*}$ by setting either $\phi=1$ or $\phi=0$. Thus it has to trade off the divergent preferences in the endorsement and failure to endorse cases. For high enough $p$, the increase in profits in the endorsement case from choosing the optimist outweigh the decrease in the failure case. For low $p$, the firm prefers the extreme pessimist who allows a large increase in price following endorsement, but who does not reduce price too much following a failure to endorse. For high $p$, the firm prefers the extreme optimist. Because $p$ is high, even a soft endorsement allows a substantial price, so the scope for a tough endorsement to raise price is less. Furthermore, the probability of the extreme optimist endorsing is very high, while a catastrophic failure to endorse becomes very unlikely. Figure 3 shows overall profits from the extreme optimist 
less those from the extreme pessimist over the range of $p$. As can be seen, choosing the better extreme increases profits of the good product firm by up to almost $4 \%$ of the maximum feasible profit (where everybody buys at price $\lambda=1$ ).

\section{Figure 3}

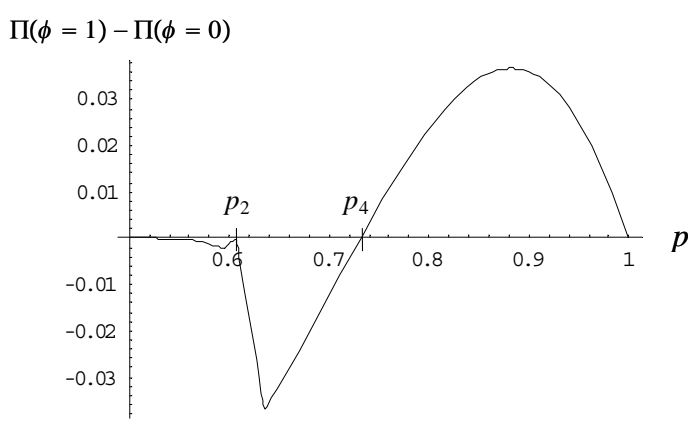

With uninformative priors, note that one interpretation of a high $p$ is of a well-established firm or a well-known type of product about which we expect consumers and reviewers to receive more precise private signals. Similarly a low $p$ might indicate a very innovative product or a new firm. Under such an interpretation, our results indicate that a new firm should seek to launch its product with a bang, hoping for an endorsement from a tough reviewer. An established firm, on the other hand, ought to be more circumspect - knowing the precision of information to be high, the good product firm has little need to take any risks.

The next two figures illustrate the magnitude of the optimal reviewer's superiority. Choosing the reviewer optimally increases profits dramatically. Figure 4 shows that the optimal reviewer increases profits of the good product firm by up to $8 \%$ over the unbiased reviewer. The optimal reviewer increases profits by up to $15 \%$ over no review, as illustrated in Figure 5.
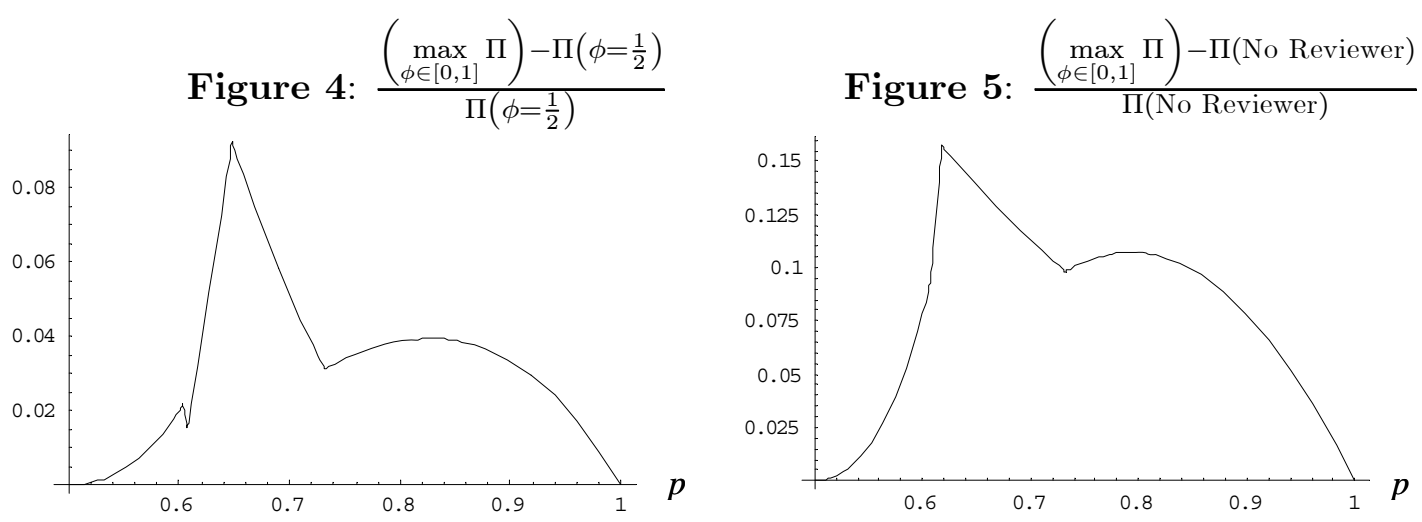


\section{Conclusion}

The results in this article provide an integration of two key choices for the producer of any new product whose quality is uncertain to consumers: the choice of an initial price, and the early product marketing strategy. Product testing and review is essential in many industries: a glance at the shelves of any news-stand will indicate how many magazines and journals provide previews of new products prior to launch. We can also think in terms of a test for a new product, where passing might involve winning a prize or achieving a hallmark or known standard of excellence, and the analysis in this paper is equally applicable when new products face tests rather than reviewers. Finally, we can also think in terms of a worker early in their career, a Ph.D. student for example, selecting a known tough or soft referee.

Quite apart from any standard signalling arguments, we have found that firms with products of quality unknown to consumers will tend towards sending their products to extremists for assessment, whether this be reviewers with known biases or tests that are publicly known to be extremely tough or soft. This is true both for a continuum of reviewer types and for a finite number: the best choice will always be either the toughest or softest test available. We have also seen that sending a product to review rather than avoiding a reviewer altogether is sensible. After the test result or reviewer's endorsement decision is known, the firm can then select an optimal price, and consumers will purchase or not based on the price, public review and their own private information. Quite remarkably, when priors are uninformative and the reviewer's and consumers' signals are not too revealing, it is the toughest reviewer who will best raise profits for the firm in expectation. This might correspond to a new or innovative firm opting for a baptism of fire, hoping for a powerful endorsement from a tough test. By enabling the firm to set a very high price following endorsement, the harsh reviewer increases expected profits while keeping the impact of a failure to endorse to a minimum. As private signals become more revealing, which might correspond to an established firm or product, the extreme optimist becomes the better choice as the likelihood of an endorsement rises, while the firm is less likely to suffer the hugely damaging impact of a soft reviewer failing to endorse.

We can therefore explain the survival of reviewers with well-known harsh styles, biases and critical approaches. While we might think firms likely to avoid such reviewers, the results in this paper show that while tough reviewers are likely to not offer any endorsement, the tremendous 
gains when they do endorse might actually make them popular with certain firms. On the other hand, the proliferation of very soft review journals, which may be supported by the very firms they seek to assess, can also be explained.

It is clear that there are many reasons why a firm might approach a reviewer, both pre-launch and post release of the product, some of which might mitigate the starkness of our findings. In order to focus on the heart of the issue, we have abstracted away from some important issues, such as inter-firm competition, advertising, the use of multiple reviewers, or the case when the firm doesn't know the quality of its own product. We might also consider how consumers learn in more complex settings, the alignment of views between certain types of consumers and reviewers, the choice of post-release reviewers by consumers, and explicitly how different types of reviewers evolve. Extending our model to incorporate any of these concerns might provide fertile ground for future research. 


\section{ApPEndix A}

In Section 4, we restricted attention to pooling equilibria in which the bad product firm is forced to follow the good product firm's choice of $\{\phi, \lambda\}$, which can be supported by the belief that any firm which deviates from the good product firm's preferences is selling a low quality product. However, as noted in footnote 11, pooling on any arbitrary $\{\phi, \lambda\}$ can also be supported by such beliefs, but we do not believe such equilibria to be reasonable. Representing a choice $\{\phi, \lambda\}$ by $x$, let $\mathcal{X}^{*}$ be the set of $x$ which are optimal for the good product firm conditional on pooling, with representative member $x^{*}$. In order to rule out pooling on $x \notin \mathcal{X}^{*}$, we need to make further refinements. One suggested refinement might be to rule out pooling on any $x$ which (conditional on pooling) is strictly Pareto dominated by at least one $x^{*}$. Now starting from a pooling equilibrium at $x$, let $\gamma(x, \widehat{x})$ represent the consumers' off-equilibrium belief about $\operatorname{Pr}[V=1]$ having seen a deviation to $\widehat{x}$. Let $\gamma^{G}(x, \widehat{x})$ and $\gamma^{B}(x, \widehat{x})$ represent the cut-off levels above which deviation from $x$ to $\widehat{x}$ is profitable for the good and bad product firms. The second refinement assumes that $\gamma(x, \widehat{x}) \geq q$ if $\gamma^{G}(x, \widehat{x}) \leq \gamma^{B}(x, \widehat{x})$ and $\gamma(x, \widehat{x}) \leq q$ if $\gamma^{G}(x, \widehat{x}) \geq \gamma^{B}(x, \widehat{x})$. Knowing that a particular type had a greater incentive to deviate to $\widehat{x}$, we might think it reasonable that consumers maintain at least the prior belief that the deviator is of that type. This refinement has the flavor of Banks and Sobel's (1987) divinity refinement. Using the refinement, we can rule out pooling on any non-dominated $x \notin \mathcal{X}^{*}$. Take any $x^{*} \in \mathcal{X}^{*}$. Because $x \notin \mathcal{X}^{*}, \gamma^{G}\left(x, x^{*}\right)<q$. But $x$ is not dominated by $x^{*}$, so $\gamma^{B}\left(x, x^{*}\right) \geq q$. Thus, $\gamma^{G}\left(x, x^{*}\right)<\gamma^{B}\left(x, x^{*}\right)$, and the refinement implies $\gamma\left(x, x^{*}\right) \geq q$. This means the good type will want to deviate, invalidating the equilibrium: the good type has a greater incentive to deviate to $x^{*}$, so consumers seeing such a deviation believe the probability of the type being good is at least $q$, giving the good type an incentive to deviate. Finally, consider pooling on any $x^{*}$. By the optimality of $x^{*}$ for the good type, $\gamma^{G}\left(x^{*}, x\right) \geq q \forall x$. For any $x$, if $\gamma^{B}\left(x^{*}, x\right) \geq \gamma^{G}\left(x^{*}, x\right)$, any $\gamma\left(x^{*}, x\right) \in\left[q, \gamma^{G}\left(x^{*}, x\right)\right]$ is consistent with our refinement and will prevent deviation. If $\gamma^{B}\left(x^{*}, x\right)<\gamma^{G}\left(x^{*}, x\right)$, then any $\gamma\left(x^{*}, x\right) \leq \min \left\{q, \gamma^{B}\left(x^{*}, x\right)\right\}$ will work. If we are content to accept a such a set of refinements, we can establish $\mathcal{X}^{*}$ as the only pooling equilibria. 


\section{Appendix B}

Proof of Lemma 1. First, note that:

$$
\begin{aligned}
\operatorname{Pr}[E] p \mu_{E, H} & =\left[p^{2}+2 p(1-p) \phi\right] p \frac{\left[p^{2}+2 p(1-p) \phi\right] p q}{\left[p^{2}+2 p(1-p) \phi\right] p q+\left[(1-p)^{2}+2 p(1-p) \phi\right](1-p)(1-q)} \\
& =\frac{p^{2} q\left[p^{2}+2 p(1-p) \phi\right]^{2}}{p^{3} q+(1-p)^{3}(1-q)+2 p(1-p) \phi[p q+(1-p)(1-q)]}
\end{aligned}
$$

Let $Z_{1}=p^{3} q+(1-p)^{3}(1-q)+2 p(1-p) \phi[p q+(1-p)(1-q)]>0$.

Then $2 p(1-p) \phi=\frac{Z_{1}-p^{3} q-(1-p)^{3}(1-q)}{p q+(1-p)(1-q)}$, so $p^{2}+2 p(1-p) \phi=\frac{Z_{1}-p^{3} q-(1-p)^{3}(1-q)+p^{2}[p q+(1-p)(1-q)]}{p q+(1-p)(1-q)}$.

Let $Y_{1}=p^{3} q+(1-p)^{3}(1-q)-p^{2}[p q+(1-p)(1-q)]=\left[(1-p)^{2}-p^{2}\right](1-p)(1-q)<0$ for $p \in\left(\frac{1}{2}, 1\right)$.

Then

$$
\operatorname{Pr}[E] p \mu_{E, H}=\frac{p^{2} q}{[p q+(1-p)(1-q)]^{2}} \frac{\left(Z_{1}-Y_{1}\right)^{2}}{Z_{1}}=\frac{p^{2} q}{[p q+(1-p)(1-q)]^{2}}\left(Z_{1}-2 Y_{1}+\frac{Y_{1}^{2}}{Z_{1}}\right)
$$

Using the fact that $\frac{d^{2} x^{-1}}{d x^{2}}=\frac{d\left(-x^{-2}\right)}{d x}=2 x^{-3}$,

$$
\frac{d^{2}\left(\operatorname{Pr}[E] p \mu_{E, H}\right)}{d Z_{1}^{2}}=\frac{p^{2} q}{[p q+(1-p)(1-q)]^{2}} \frac{2 Y_{1}^{2}}{Z_{1}^{3}}>0
$$

Thus, using $\frac{d Z_{1}}{d \phi}=2 p(1-p)[p q+(1-p)(1-q)]>0$,

$$
\frac{d^{2}\left(\operatorname{Pr}[E] p \mu_{E, H}\right)}{d \phi^{2}}=\frac{d\left(\frac{d\left(\operatorname{Pr}[E] p \mu_{E, H}\right)}{d Z_{1}} \frac{d Z_{1}}{d \phi}\right)}{d \phi}=\frac{d^{2}\left(\operatorname{Pr}[E] p \mu_{E, H}\right)}{d Z_{1}^{2}}\left(\frac{d Z_{1}}{d \phi}\right)^{2}>0
$$

Second, note that:

$$
\begin{aligned}
\operatorname{Pr}[E] \mu_{E, L} & =\left[p^{2}+2 p(1-p) \phi\right] \frac{\left[p^{2}+2 p(1-p) \phi\right](1-p) q}{\left[p^{2}+2 p(1-p) \phi\right](1-p) q+\left[(1-p)^{2}+2 p(1-p) \phi\right] p(1-q)} \\
& =\frac{(1-p) q\left[p^{2}+2 p(1-p) \phi\right]^{2}}{p^{2}(1-p) q+(1-p)^{2} p(1-q)+2 p(1-p) \phi[(1-p) q+p(1-q)]}
\end{aligned}
$$

Letting $Z_{2}=p^{2}(1-p) q+(1-p)^{2} p(1-q)+2 p(1-p) \phi[(1-p) q+p(1-q)]>0$ and $Y_{2}=$ $p^{2}(1-p) q+(1-p)^{2} p(1-q)-p^{2}[(1-p) q+p(1-q)]=\left[(1-p)^{2}-p^{2}\right] p(1-q)<0$, and noting that $\frac{d Z_{2}}{d \phi}>0$, the proof proceeds along the same lines as in the first case. 
Third, note that:

$$
\begin{aligned}
& \operatorname{Pr}[F] p \mu_{F, H} \\
= & {\left[(1-p)^{2}+2 p(1-p)(1-\phi)\right] p \frac{\left[(1-p)^{2}+2 p(1-p)(1-\phi)\right] p q}{\frac{p^{2} q\left[(1-p)^{2}+2 p(1-p)(1-\phi)\right]^{2}}{(1-2 p(1-p)(1-\phi)] p q+\left[p^{2}+2 p(1-p)(1-\phi)\right](1-p)(1-q)}} } \\
= & \frac{{ }^{2} p q+p^{2}(1-p)(1-q)+2 p(1-p)(1-\phi)[p q+(1-p)(1-q)]}{}
\end{aligned}
$$

Letting $Z_{3}=(1-p)^{2} p q+p^{2}(1-p)(1-q)+2 p(1-p)(1-\phi)[p q+(1-p)(1-q)]>0$ and $Y_{3}=(1-p)^{2} p q+p^{2}(1-p)(1-q)-(1-p)^{2}[p q+(1-p)(1-q)]=\left[p^{2}-(1-p)^{2}\right](1-p)(1-q)>0$, and noting that $\frac{d Z_{3}}{d \phi}<0$, the proof proceeds along the same lines as in the first case.

Fourth, note that:

$$
\begin{aligned}
& \operatorname{Pr}[F] \mu_{F, L} \\
= & {\left[(1-p)^{2}+2 p(1-p)(1-\phi)\right] \frac{\left[(1-p)^{2}+2 p(1-p)(1-\phi)\right](1-p) q}{\left[(1-p)^{2}+2 p(1-p)(1-\phi)\right](1-p) q+\left[p^{2}+2 p(1-p)(1-\phi)\right] p(1-q)} } \\
= & \frac{(1-p) q\left[(1-p)^{2}+2 p(1-p)(1-\phi)\right]^{2}}{(1-p)^{3} q+p^{3}(1-q)+2 p(1-p)(1-\phi)[(1-p) q+p(1-q)]}
\end{aligned}
$$

Letting $Z_{4}=(1-p)^{3} q+p^{3}(1-q)+2 p(1-p)(1-\phi)[(1-p) q+p(1-q)]>0$ and $Y_{4}=$ $(1-p)^{3} q+p^{3}(1-q)-(1-p)^{2}[(1-p) q+p(1-q)]=\left[p^{2}-(1-p)^{2}\right] p(1-q)>0$, and noting that $\frac{d Z_{4}}{d \phi}<0$, the proof proceeds along the same lines as in the first case.

Proof of Lemma 2. (i) $\Pi_{H H}\left(\phi=\frac{1}{2}\right)>p \mu_{H}$

Given $\phi=\frac{1}{2}$ and using (1) and (4),

$$
\begin{aligned}
\operatorname{Pr}[E] p \mu_{E, H} & =\frac{p^{4} q}{p^{2} q+(1-p)^{2}(1-q)} \\
\operatorname{Pr}[F] p \mu_{F, H} & =(1-p) p \frac{(1-p) p q}{(1-p) p q+p(1-p)(1-q)}=p(1-p) q
\end{aligned}
$$

while

$$
p \mu_{H}=\frac{p^{2} q}{p q+(1-p)(1-q)}
$$

Thus,

$$
\Pi_{H H}\left(\phi=\frac{1}{2}\right)-p \mu_{H}=\frac{p^{4} q}{p^{2} q+(1-p)^{2}(1-q)}+p(1-p) q-\frac{p^{2} q}{p q+(1-p)(1-q)}
$$

Dividing through by $p q>0$ and multiplying by $\left[p^{2} q+(1-p)^{2}(1-q)\right][p q+(1-p)(1-q)]>$ 
$0, \Pi_{H H}\left(\phi=\frac{1}{2}\right)-p \mu_{H}$ has the same sign as

$$
1-2 q+q^{2}-5 p+8 p^{2}-4 p^{3}+10 p q-5 p q^{2}-16 p^{2} q+8 p^{2} q^{2}+8 p^{3} q-4 p^{3} q^{2}
$$

which factors to $(1-p)(2 p-1)^{2}(1-q)^{2}>0$.

(ii) $\Pi_{L L}\left(\phi=\frac{1}{2}\right)>\mu_{L}$

Given $\phi=\frac{1}{2}$ and using (3) and (5),

$$
\begin{aligned}
\operatorname{Pr}[E] \mu_{E, L} & =p \frac{p(1-p) q}{p(1-p) q+(1-p) p(1-q)}=p q \\
\operatorname{Pr}[F] \mu_{F, L} & =\frac{(1-p)^{3} q}{(1-p)^{2} q+p^{2}(1-q)}
\end{aligned}
$$

while

$$
\mu_{L}=\frac{(1-p) q}{(1-p) q+p(1-q)}
$$

Thus,

$$
\Pi_{L L}\left(\phi=\frac{1}{2}\right)-\mu_{L}=p q+\frac{(1-p)^{3} q}{(1-p)^{2} q+p^{2}(1-q)}-\frac{(1-p) q}{(1-p) q+p(1-q)}
$$

Dividing through by $q>0$ and multiplying by $\left[(1-p)^{2} q+p^{2}(1-q)\right][(1-p) q+p(1-q)]>0$, $\Pi_{L L}\left(\phi=\frac{1}{2}\right)-\mu_{L}$ has the same sign as

$$
p-4 p^{2}+4 p^{3}-2 p q+p q^{2}+8 p^{2} q-4 p^{2} q^{2}-8 p^{3} q+4 p^{3} q^{2}
$$

which factors to $p(2 p-1)^{2}(1-q)^{2}>0$.

Proof of Lemma 3. First, note that given $q=\frac{1}{2}$, and using (2), (3), (4) and (5),

$$
\begin{gathered}
\operatorname{Pr}[E] p \mu_{E, H}=\frac{p^{2}\left[p^{2}+2 p(1-p) \phi\right]^{2}}{p^{3}+(1-p)^{3}+2 p(1-p) \phi} \\
\operatorname{Pr}[E] \mu_{E, L}=\frac{\left[p^{2}+2 p(1-p) \phi\right]^{2}(1-p)}{\left[p^{2}+2 p(1-p) \phi\right](1-p)+\left[(1-p)^{2}+2 p(1-p) \phi\right] p}=\frac{\left[p^{2}+2 p(1-p) \phi\right]^{2}(1-p)}{p(1+2 \phi)(1-p)} \\
\operatorname{Pr}[F] p \mu_{F, H}=\frac{p^{2}\left[(1-p)^{2}+2 p(1-p)(1-\phi)\right]^{2}}{\left[(1-p)^{2}+2 p(1-p)(1-\phi)\right] p+\left[p^{2}+2 p(1-p)(1-\phi)\right](1-p)}=\frac{p^{2}\left[(1-p)^{2}+2 p(1-p)(1-\phi)\right]^{2}}{p(1-p)(3-2 \phi)} \\
\operatorname{Pr}[F] \mu_{F, L}=\frac{\left[(1-p)^{2}+2 p(1-p)(1-\phi)\right]^{2}(1-p)}{\left[(1-p)^{2}+2 p(1-p)(1-\phi)\right](1-p)+\left[p^{2}+2 p(1-p)(1-\phi)\right] p}=\frac{\left[(1-p)^{2}+2 p(1-p)(1-\phi)\right]^{2}(1-p)}{p^{2}+(1-p)-2 p(1-p) \phi}
\end{gathered}
$$


Now, $p \mu_{d, H} \geq \mu_{d, L} \Leftrightarrow \frac{p \mu_{d, H}}{\mu_{d, L}} \geq 1$. Using (8) and (9):

$$
\frac{p \mu_{F, H}}{\mu_{F, L}}=\frac{p\left[p^{2}+(1-p)-2 p(1-p) \phi\right]}{(1-p)^{2}(3-2 \phi)}
$$

Where $\phi=1,(10) \geq 1$ iff

$$
p^{3}+p-p^{2}-2 p^{2}+2 p^{3} \geq 1-2 p+p^{2} \Leftrightarrow 3 p^{3}-4 p^{2}+3 p-1 \geq 0
$$

$\frac{d\left(3 p^{3}-4 p^{2}+3 p-1\right)}{d p}=9 p^{2}-8 p+3=p^{2}-8 p(1-p)+3>0$ given $p(1-p)<\frac{1}{4}$. Solving numerically, we find that $3 p^{3}-4 p^{2}+3 p-1=0$ at $p_{1} \simeq 0.594$, proving (i).

Where $\phi=0,(10) \geq 1$ iff

$$
p^{3}+p-p^{2} \geq 3-6 p+3 p^{2} \Leftrightarrow p^{3}-4 p^{2}+7 p-3 \geq 0
$$

$\frac{d\left(p^{3}-4 p^{2}+7 p-3\right)}{d p}=3 p^{2}-8 p+7=-3 p(1-p)-5 p+7>0$ given $p(1-p)<\frac{1}{4}$. Solving numerically, $p^{3}-4 p^{2}+7 p-3=0$ at $p_{2} \simeq 0.607$, proving (ii).

Using (6) and (7):

$$
\frac{p \mu_{E, H}}{\mu_{E, L}}=\frac{p^{2}(2 p \phi+p)}{p^{3}+(1-p)^{3}+2 p(1-p) \phi}
$$

Where $\phi=1,(11) \geq 1$ iff

$$
3 p^{3} \geq p^{3}+(1-p)\left(1+p^{2}\right) \Leftrightarrow 3 p^{3}-p^{2}+p-1 \geq 0
$$

$\frac{d\left(3 p^{3}-p^{2}+p-1\right)}{d p}=9 p^{2}-2 p+1=7 p^{2}-2 p(1-p)+1>0$ given $p(1-p)<\frac{1}{4}$. Solving numerically, $3 p^{3}-p^{2}+p-1=0$ at $p_{3} \simeq 0.635$, proving (iii) ${ }^{15}$

Where $\phi=0,(11) \geq 1$ iff $p^{3} \geq p^{3}+(1-p)^{3}$ which can never be true, proving (iv).

15 The values of $p_{1}, p_{2}$ and $p_{3}$ can all be found analytically, but the expressions are voluminous and doing so does not add anything to the argument. 
Proof of Remark 2. Let $Q=p(1-p)(3-2 \phi)$ and $R=p(1-p)(1+2 \phi)$. Note that $Q \in(0,1)$ and $R \in(0,1)$ as $p(1-p) \in\left(0, \frac{1}{4}\right)$. Note also that:

$$
\begin{aligned}
p^{2}+2 p(1-p) \phi & =(1-p)(2 p-1)+(1-Q)=p(2 p-1)+R \\
p^{3}+(1-p)^{3}+2 p(1-p) \phi & =1-Q \\
(1-p)^{2}+2 p(1-p)(1-\phi) & =(1-p)(1-2 p)+Q=p(1-2 p)+(1-R) \\
p^{2}+(1-p)-2 p(1-p) \phi & =1-R
\end{aligned}
$$

Then, using (6) to (9),

$$
\begin{gathered}
\operatorname{Pr}[E] p \mu_{E, H}=\frac{p^{2}(1-p)^{2}(2 p-1)^{2}}{1-Q}+2 p^{2}(1-p)(2 p-1)+p^{2}(1-Q) \\
\operatorname{Pr}[E] \mu_{E, L}=\frac{(1-p) p^{2}(2 p-1)^{2}}{R}+2(1-p) p(2 p-1)+(1-p) R \\
\operatorname{Pr}[F] p \mu_{F, H}=\frac{p^{2}(1-p)^{2}(1-2 p)^{2}}{Q}+2 p^{2}(1-p)(1-2 p)+p^{2} Q \\
\operatorname{Pr}[F] \mu_{F, L}=\frac{(1-p) p^{2}(1-2 p)^{2}}{1-R}+2(1-p) p(1-2 p)+(1-p)(1-R)
\end{gathered}
$$

We consider four ranges in turn: (i) $p \in\left(\frac{1}{2}, p_{1}\right)$; (ii) $\left[p_{1}, p_{2}\right)$; (iii) $\left[p_{2}, p_{3}\right)$; (iv) $\left[p_{3}, 1\right.$ ).

(i) From Lemma $3, \Pi=\Pi_{L L}=\operatorname{Pr}[E] \mu_{E, L}+\operatorname{Pr}[F] \mu_{F, L}$ for $p \in\left(\frac{1}{2}, p_{1}\right)$. From (13) and (15),

$$
\Pi_{L L}=\frac{(1-p) p^{2}(2 p-1)^{2}}{R(1-R)}+(1-p)
$$

Therefore,

$$
\begin{aligned}
\Pi_{L L}(\phi=0) & >\Pi_{L L}(\phi=1) \Leftrightarrow \\
R(\phi=0)[1-R(\phi=0)] & <R(\phi=1)[1-R(\phi=1)] \Leftrightarrow \\
{[p(1-p)][1-p(1-p)] } & <[3 p(1-p)][1-3 p(1-p)] \Leftrightarrow p(1-p)<\frac{1}{4}
\end{aligned}
$$

which holds given $p \in\left(\frac{1}{2}, 1\right)$. 
(ii) Let $\Pi_{L H}=\operatorname{Pr}[E] \mu_{E, L}+\operatorname{Pr}[F] p \mu_{F, H}$. From Lemma 3, $\Pi(\phi=0)=\Pi_{L L}(\phi=0)$ and $\Pi(\phi=1)=\Pi_{L H}(\phi=1)$ for $p \in\left[p_{1}, p_{2}\right)$. Using (7) and (9):

$$
\Pi_{L L}(\phi=0)=\frac{\left[p^{2}\right]^{2}(1-p)}{p(1-p)}+\frac{\left[(1-p)^{2}+2 p(1-p)\right]^{2}(1-p)}{\left[p^{2}+(1-p)\right]}=\frac{1-p-2 p^{2}+3 p^{3}}{1-p+p^{2}}
$$

while using (7) and (8):

$$
\Pi_{L H}(\phi=1)=\frac{\left[p^{2}+2 p(1-p)\right]^{2}(1-p)}{3 p(1-p)}+\frac{p^{2}\left[(1-p)^{2}\right]^{2}}{p(1-p)}=\frac{7 p-13 p^{2}+10 p^{3}-3 p^{4}}{3}
$$

Thus:

$$
\begin{aligned}
\Pi_{L L}(\phi=0) & >\Pi_{L H}(\phi=1) \Leftrightarrow \\
3-3 p-6 p^{2}+9 p^{3}-\left(1-p+p^{2}\right)\left(7 p-13 p^{2}+10 p^{3}-3 p^{4}\right) & >0 \Leftrightarrow \\
3-10 p+14 p^{2}-21 p^{3}+26 p^{4}-13 p^{5}+3 p^{6} & >0 \Leftrightarrow \\
\left(-3+7 p-4 p^{2}+p^{3}\right)\left(-1+p-p^{2}+3 p^{3}\right) & >0
\end{aligned}
$$

From part (ii) of the proof of Lemma $3,-3+7 p-4 p^{2}+p^{3}<0$ here. From part (iii) of the same proof, $-1+p-p^{2}+3 p^{3}<0$ here also.

(iii) From Lemma $3, \Pi=\Pi_{L H}$ for $p \in\left[p_{2}, p_{3}\right)$. Using (7) and (8):

$$
\Pi_{L H}(\phi=0)=p^{3}+\frac{p^{2}\left[(1-p)^{2}+2 p(1-p)\right]^{2}}{3 p(1-p)}=\frac{p+p^{2}+2 p^{3}-p^{4}}{3}
$$

Thus, using (16),

$$
\begin{aligned}
\Pi_{L H}(\phi=0) & \gtreqless \Pi_{L H}(\phi=1) \Leftrightarrow \\
p+p^{2}+2 p^{3}-p^{4} & \gtreqless 7 p-13 p^{2}+10 p^{3}-3 p^{4} \Leftrightarrow \\
-3+7 p-4 p^{2}+p^{3} & \gtreqless 0
\end{aligned}
$$

From part (ii) of the proof of Lemma $3,-3+7 p-4 p^{2}+p^{3}>0$ here, except at $p_{2}$ where it equals zero. 
(iv) Remember that $\Pi_{H H}=\operatorname{Pr}[E] p \mu_{E, H}+\operatorname{Pr}[F] p \mu_{F, H}$. From Lemma $3, \Pi(\phi=0)=\Pi_{L H}(\phi=0)$ and $\Pi(\phi=1)=\Pi_{H H}(\phi=1)$ for $\left[p_{3}, 1\right)$. From (12) and (14), and using the fact that $Q(\phi=1)=$ $p(1-p)$,

$$
\begin{aligned}
\Pi_{H H} & =\frac{p^{2}(1-p)^{2}(2 p-1)^{2}}{Q(1-Q)}+p^{2} \\
& \therefore \quad \Pi_{H H}(\phi=1)=\frac{p(1-p)(2 p-1)^{2}}{1-p+p^{2}}+p^{2}=\frac{p-5 p^{2}+8 p^{3}-4 p^{4}}{1-p+p^{2}}+p^{2}
\end{aligned}
$$

Thus, using (17),

$$
\begin{aligned}
\Pi_{L H}(\phi=0) & \gtreqless \Pi_{H H}(\phi=1) \Leftrightarrow \\
\left(p+p^{2}+2 p^{3}-p^{4}-3 p^{2}\right)\left(1-p+p^{2}\right) & \gtreqless 3\left(p-5 p^{2}+8 p^{3}-4 p^{4}\right) \Leftrightarrow \\
-2 p+12 p^{2}-19 p^{3}+7 p^{4}+3 p^{5}-p^{6} & \gtreqless 0 \Leftrightarrow \\
p(1-p)\left(-1+4 p-p^{2}\right)\left(2-2 p-p^{2}\right) & \gtreqless 0 \Leftrightarrow
\end{aligned}
$$

Clearly, $p(1-p)>0$. Also, $-1+4 p-p^{2}=3 p+p(1-p)-1>0$ for $p>\frac{1}{2}$. As $-2-2 p<0$, $2-2 p-p^{2}$ is strictly decreasing for $p>\frac{1}{2}$, and equals zero at $p_{4}=\frac{2-\sqrt{4-(-8)}}{-2}=-1+\sqrt{3} \simeq 0.732$. Thus, we conclude that for $p \in\left[p_{3}, p_{4}\right), \Pi_{L H}(\phi=0)>\Pi_{H H}(\phi=1)$, for $p \in\left(p_{4}, 1\right), \Pi_{L H}(\phi=0)<$ $\Pi_{H H}(\phi=1)$, and at $p_{4}, \Pi_{L H}(\phi=0)=\Pi_{H H}(\phi=1)$. 


\section{Appendix C}

Here we show how Propositions 1 and 2 extend to the more general case where $V \in\{\underline{V}, \bar{V}\}$ and there is a constant marginal cost of production $c$ with $0 \leq c \leq \underline{V}<\bar{V}$, as claimed in footnote 5 .

In the general case, a consumer with belief $\mu=\operatorname{Pr}[V=\bar{V}]$ will be willing to pay

$$
\mu \bar{V}+(1-\mu) \underline{V}=\mu(\bar{V}-\underline{V})+\underline{V}
$$

Thus,

$$
\pi_{d}^{*}=\max \left\{p \mu_{d, H}(\bar{V}-\underline{V})+p \underline{V}-p c, \mu_{d, L}(\bar{V}-\underline{V})+\underline{V}-c\right\}
$$

Now, $\frac{d^{2}\left(\operatorname{Pr}[d]\left(p \mu_{d, H}(\bar{V}-\underline{V})+p \underline{V}-p c\right)\right)}{d \phi^{2}}=\frac{d^{2}\left(\operatorname{Pr}[d] p \mu_{d, H}\right)}{d \phi^{2}}(\bar{V}-\underline{V})>0$ using Lemma 1 and the fact that $\operatorname{Pr}[E]=p^{2}+2 p(1-p) \phi$ and $\operatorname{Pr}[F]=(1-p)^{2}+2 p(1-p)(1-\phi)$ are linear in $\phi$. Similarly, $\frac{d^{2}\left(\operatorname{Pr}[d]\left(\mu_{d, L}(\bar{V}-\underline{V})+\underline{V}-c\right)\right)}{d \phi^{2}}=\frac{d^{2}\left(\operatorname{Pr}[d] \mu_{d, L}\right)}{d \phi^{2}}(\bar{V}-\underline{V})>0$. The proof of Proposition 1 then proceeds on the same lines as before.

With regard to Proposition 2,

$$
\begin{aligned}
\Pi_{H H}= & \operatorname{Pr}[E]\left(p \mu_{E, H}(\bar{V}-\underline{V})+p \underline{V}-p c\right)+\operatorname{Pr}[F]\left(p \mu_{F, H}(\bar{V}-\underline{V})+p \underline{V}-p c\right) \\
= & \left(\operatorname{Pr}[E] p \mu_{E, H}+\operatorname{Pr}[F] p \mu_{F, H}\right)(\bar{V}-\underline{V})+p \underline{V}-p c \\
\Pi_{L L}= & \operatorname{Pr}[E]\left(\mu_{E, L}(\bar{V}-\underline{V})+\underline{V}-c\right)+\operatorname{Pr}[F]\left(\mu_{F, L}(\bar{V}-\underline{V})+\underline{V}-c\right) \\
= & \left(\operatorname{Pr}[E] \mu_{E, L}+\operatorname{Pr}[F] \mu_{F, L}\right)(\bar{V}-\underline{V})+\underline{V}-c
\end{aligned}
$$$$
\Pi(\text { No Reviewer })=\max \left\{p \mu_{H}(\bar{V}-\underline{V})+p \underline{V}-p c, \mu_{L}(\bar{V}-\underline{V})+\underline{V}-c\right\}
$$

Thus, $\Pi_{H H}>p \mu_{H}(\bar{V}-\underline{V})+p \underline{V}-p c \Leftrightarrow \operatorname{Pr}[E] p \mu_{E, H}+\operatorname{Pr}[F] p \mu_{F, H}>p \mu_{H}$, which holds for $\phi=\frac{1}{2}$ from the proof of Lemma 2. Similarly, $\Pi_{L L}>\mu_{L}(\bar{V}-\underline{V})+\underline{V}-c$ for $\phi=\frac{1}{2}$. The proof then proceeds just as before. 


\section{REFERENCES}

Albano, G. L. and Lizzeri, A. (2001) 'Strategic Certification and Provision of Quality', International Economic Review, 42, 267-283.

Banks, J. S. and Sobel, J. (1987) 'Equilibrium Selection in Signaling Games', Econometrica $55,647-661$.

Bose, S., Orosel, G., Ottaviani, M. and Vesterlund, L. (2005a) 'Dynamic Monopoly Pricing with Herding', Discussion paper No. 5003. Centre for Economic Policy Research. Forthcoming in RAND Journal of Economics.

Bose, S., Orosel, G., Ottaviani, M. and Vesterlund, L. (2005b) 'Monopoly Pricing in the Binary Herding Model', Mimeo.

Calvert, R. L. (1985) 'The Value of Biased Information: A Rational Choice Model of Political Advice', Journal of Politics, 47, 530-555.

Caminal, R. and Vives, X. (1996) 'Why Market Shares Matter: An Information-Based Theory', RAND Journal of Economics, 27, 221-239.

Caminal, R. and Vives, X. (1999) 'Price Dynamics and Consumer Learning', Journal of Economics and Management Strategy, 8, 95-131.

Chamley, C. P. (2004) Rational Herds: Economic Models of Social Learning, Cambridge University Press.

Chiao, B., Lerner, J. and Tirole, J. (2005) 'The Rules of Standard Setting Organizations: An Empirical Analysis', NBER Working Paper 11156.

De Vany, A. and Walls, W. D. (1996) 'Bose-Einstein Dynamics and Adaptive Contracting in the Motion Picture Industry', Economic Journal, 106, 1493-1514.

Farhi, E., Lerner, J. and Tirole J. (2005) 'Certifying New Technologies', Journal of the European Economic Association, 3, 734-744.

Gill, D. and Sgroi, D. (2003) 'Product Launches with Biased Reviewers: The Importance of Not Being Earnest', Cambridge Working Papers in Economics 0334.

Gill, D. and Sgroi, D. (2006) 'Sequential Decisions with Tests'. Forthcoming in Games and Economic Behavior [corrected proof available on GEB website].

Judd, K. L. and Riordan, M. (1994) 'Price and Quality in a New Product Monopoly', Review of Economic Studies, 61, 773-789. 
Lerner, J. and Tirole, J. (2006) 'A Model of Forum Shopping', American Economic Review, 96, 1091-1113.

Lizzeri, A. (1999) 'Information Revelation and Certification Intermediaries', RAND Journal of Economics, 30, 214-231.

Mahenc, P. (2004) 'Influence of Informed Buyers in Markets Susceptible to the Lemons Problem', American Journal of Agricultural Economics, 86, 649-659.

Milgrom, P. R. (1981), 'Good News and Bad News: Representation Theorems and Applications', Bell Journal of Economics, 12, 380-391.

Ottaviani, M. and Prat, A. (2001) 'The Value of Public Information in Monopoly', Econometrica, 69, 1673-1683.

Robinson, J. (1999), The Oxford Companion to Wine (2nd ed.), Oxford University Press.

Rockafellar, R. T. (1970) Convex Analysis, Princeton University Press.

Sah, R. K. and Stiglitz, J. E. (1986), 'The Architecture of Economic Systems: Hierarchies and Polyarchies', American Economic Review, 76, 716-727.

Sgroi, D. (2002) 'Optimizing Information in the Herd: Guinea Pigs, Profits and Welfare', Games and Economic Behavior, 39, 137-166.

Taylor, C. R. (1999) 'Time-on-the-Market as a Sign of Quality', Review of Economic Studies, $66,555-578$.

Welch, I. (1992) 'Sequential Sales, Learning, and Cascades, Journal of Finance, 47, 695-732. 